Paper

\title{
Analog CMOS circuit implementation of a pulse-coupled phase oscillator system and observation of synchronization phenomena
}

\author{
Kenji Matsuzaka ${ }^{1}$, Takashi Tohara ${ }^{1}$, Kazuki Nakada ${ }^{1}$, \\ and Takashi Morie ${ }^{1 a)}$ \\ ${ }^{1}$ Graduate School of Life Science and Systems Engineering, \\ Kyushu Institute of Technology, Kitakyushu 808-0196, Japan \\ a) morie@brain.kyutech.ac.jp
}

Received August 20, 2011; Revised November 28, 2011; Published April 1, 2012

\begin{abstract}
Analog CMOS circuit implementation of a system of pulse-coupled phase oscillators is proposed. A CMOS circuit that achieves the dynamics of pulse-coupled oscillators has been designed and fabricated using a $0.25-\mu \mathrm{m}$ CMOS technology. The proposed oscillator circuits with continuous-time operation interact with each other via a pulse at each firing time. Update of the oscillator state is achieved by integrating the phase sensitivity function with the pulse width time span. The phase sensitivity function is generated by the combination of binary functions, while the function consists of three-values $\{-1,0,1\}$. Introducing a zero-value span in the function leads to fast synchronization and robustness to parameter fluctuation due to LSI device mismatches, which facilitates VLSI implementation. Using the fabricated CMOS circuit, we have observed not only in- and anti-phase but also out-of-phase synchronization.
\end{abstract}

Key Words: analog CMOS circuit, pulse-coupled phase oscillator, synchronization

\section{Introduction}

Collective synchronization phenomena occur ubiquitously in nature. Such phenomena can be modeled by ensembles of nonlinear limit-cycle oscillators. In general, an ensemble of coupled limit-cycle oscillators can be reduced into a system of coupled phase oscillators by means of the phase reduction technique [1-5].

Coupled phase oscillators are classified into two types: phase-coupled and pulse-coupled oscillators, according to the interaction among the oscillators. In the conventional theories about coupled phase oscillators, mutual interactions among phase oscillators are represented as a phase coupling function, which is a function of the phase difference between two oscillators $[1,2]$.

In order to realize large-scale coupled oscillator systems for various practical applications, CMOS LSI implementation of coupled phase oscillator systems is essential. In our previous work, a CMOS 
circuit for the phase-coupled oscillators has been proposed [6] by using the pulse-width and pulsephase modulation (PWM/PPM) approaches [7]. We applied the circuit technique to phase-locked loop neural networks [8] and visual image processing [9]. The circuit can realize functions of phase-coupled oscillators with an arbitrary phase coupling function. However, it has limitations in the operation speed and efficiency due to discrete-time operation based on the external clocks [6]. To overcome these limitations, we have proposed an analog CMOS circuit for pulse-coupled oscillators based on continuous-time operation [10].

There has been earlier work about analog CMOS circuit implementation of pulse-coupled oscillators [11-13]. They targeted at applications of communication devices, and achieved fast synchronization with low power consumption. However, the aim of this work is to achieve various synchronization phenomena, such as in-phase, anti-phase, and out-of-order synchronization, by using a simple phase sensitivity function, which leads to simple circuit implementation. A promising application of our system is image processing [14].

In this paper, we describe the analog CMOS circuit for pulse-coupled oscillators in detail. We designed and fabricated the circuit using a $0.25-\mu \mathrm{m}$ CMOS technology. We demonstrate that the circuit exhibits in-/anti-phase and different (out-of-phase) synchronization through experiments using the fabricated circuit.

\section{Pulse-coupled phase oscillator model}

The concept of coupled phase oscillators has been firstly proposed by Winfree [3] and its dynamics is expressed as follows:

$$
\frac{d \phi_{i}}{d t}=\omega_{i}+Z\left(\phi_{i}\right) S(t),
$$

where $\phi_{i}$ is the $i$-th phase variable with $2 \pi$ periodicity, $\omega_{i}$ the $i$-th natural angular frequency, $Z\left(\phi_{i}\right)$ the phase sensitivity function, which gives the response of the $i$-th oscillator, and $S(t)$ the summation of inputs from other oscillators. We assume here the pulse inputs as follows:

$$
S(t)=\frac{K_{0}}{N} \sum_{j=1}^{N} \sum_{n=1}^{\infty} \delta\left(t-t_{j n}\right)
$$

where $K_{0}$ the coupling strength, $N$ the number of oscillators, and $t_{j n}$ a firing time. Function $\delta$ is mathematically Dirac's delta function, and only represents the input spike timing without a pulse width. However, in the real circuit, a spike pulse has a definite width $\Delta t$, during which $\phi_{i}$ is updated according to the value of $Z\left(\phi_{i}\right)$. If it is assumed that $Z\left(\phi_{i}\right)=-\sin \left(\phi_{i}\right)$ as a specific case, the dynamics of the pulse-coupled oscillators are schematically illustrated in Fig. 1.

Pulse-coupled oscillators may be transformed into a canonical form of phase-coupled oscillators [15]. By replacing $t-t_{j n}=\left[\phi_{j}(t)-2 \pi n\right] / \omega_{0}$ and averaging in time, we can obtain the following equivalent dynamics [1]:

$$
\frac{d \phi_{i}}{d t}=\omega_{i}-\frac{K}{N} \sum_{j=1}^{N} \sin \left(\phi_{i}-\phi_{j}\right)
$$

where $K=2 \pi K_{0} / \omega_{0}$. This dynamical equation is widely known as the Kuramoto model [1,2].

Despite of this approximate equivalence in theoretical analysis between pulse-coupled and phasecoupled oscillator models, from a hardware implementation point of view, pulse-coupled systems are more suitable than phase-coupled systems. In the latter systems, information transported between oscillators is represented by analog values, which must be buffered by analog circuits, and this leads to less efficiency and more power consumption of the systems. On the other hand, in the former systems, information is represented by pulses, which can be buffered by digital circuits.

In our system, we use three-value functions as $Z\left(\phi_{i}\right)$; a typical function shape is shown in Fig. 2 . The three-value functions are expanded in the time domain and have values: $-1,0,1$. In the update operation, " -1 " and "1" correspond to decrease and increase of $\phi_{i}$, respectively, and "0" means no updating. Increasing $\phi_{i}$ results in leading of next spike firing timing and vice versa. 


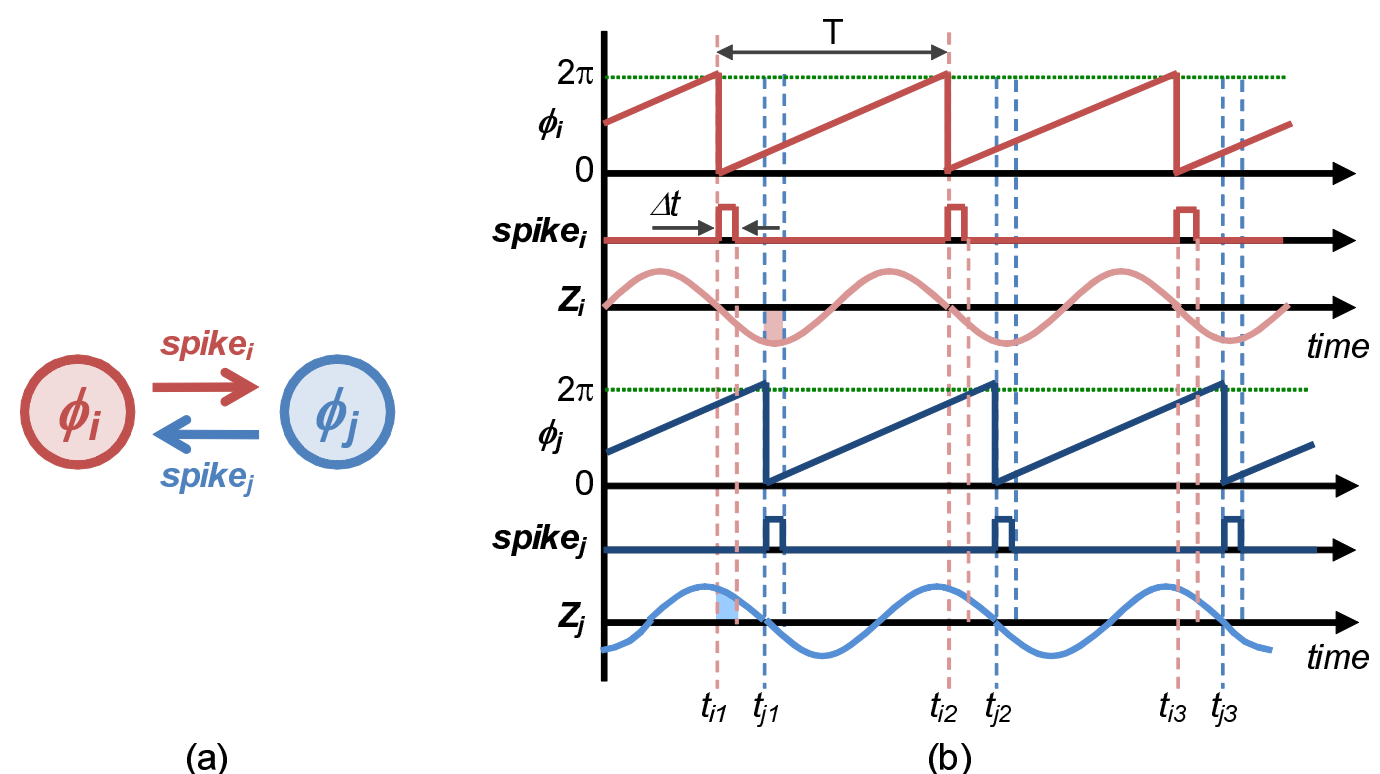

(a)

(b)

Fig. 1. Dynamics of pulse-coupled phase oscillator model.

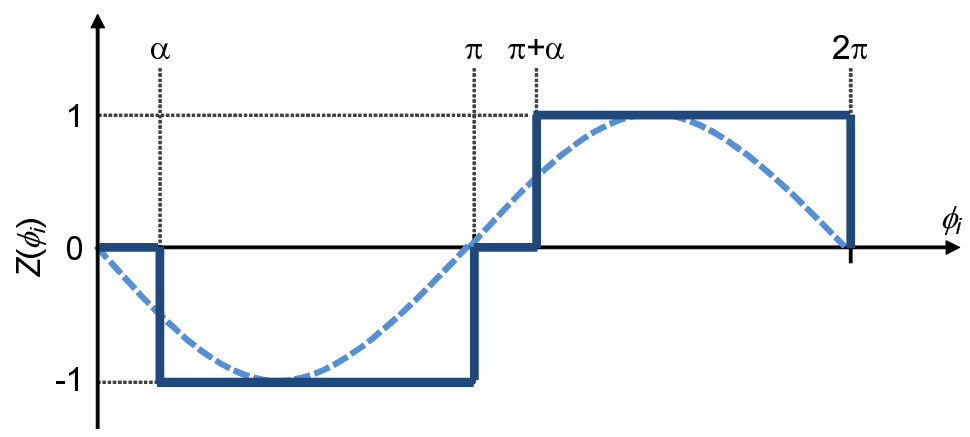

Fig. 2. An example of the shape of function $Z\left(\phi_{i}\right)$.

We newly introduce parameter $\alpha$ as the span during which $Z\left(\phi_{i}\right)=0$, as shown in Fig. 2. By setting $\alpha>\Delta t$, we can prevent the connected oscillators from over-updating when they approach to a synchronization state. Even in such a case, if $Z\left(\phi_{i}\right)$ is like that shown in Fig. 2, either oscillator is updated, and finally the system reach a synchronization state. Compared with sinusoidal functions, the three-value functions lead to faster convergence and simpler circuit implementation.

\section{CMOS circuit for pulse-coupled oscillator systems}

The oscillator unit circuit consists of oscillator $O S C$, phase sensitivity function generator $Z G E N$, and phase state updater $U P D$, as shown in Fig. 3.

\subsection{Oscillator circuit}

Figure 4 shows the oscillator circuit, which stores the phase variable of the oscillator. The phase variable is converted into spike pulses by thresholding operation using comparators, and output spike pulses to other oscillators. The circuit consists of a capacitor, two switched current sources, two comparators each of which is followed by a pulse generator $P G$, and a reset switch, as shown in Fig. 4(a). The capacitor stores a charge as the phase variable, and switched current source $I_{\omega}$ charges a constant charge to the capacitor for realizing $\omega_{i}$ in Eq. (1). Switched current source $I_{\text {set }}$ sets the initial phase state by using a PWM signal given from terminal input. Two sets of comparators comp shown in Fig. 4(b) and pulse generators $P G$ shown in Fig. 4(c) are used for generating spike outputs, spike and reset.

The timing diagram for circuit operation is shown in Fig. 4(d). The terminal voltage of the capacitor, $V_{\phi}$, increases continuously by the current $I_{\omega}$ from the current source. When $V_{\phi}$ reaches constant 


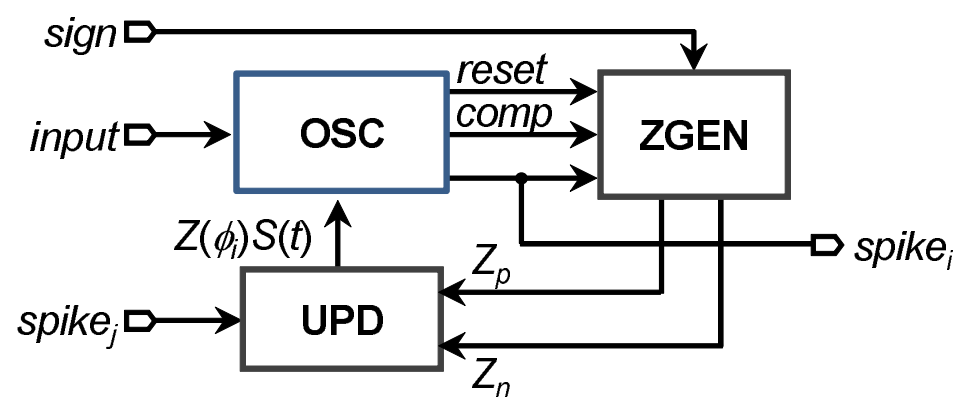

Fig. 3. Oscillator unit circuit architecture.

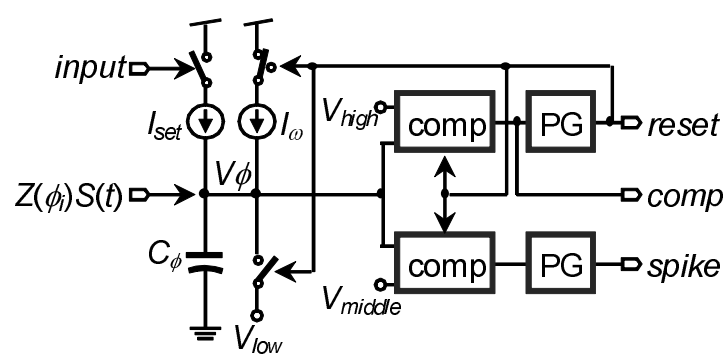

(a)

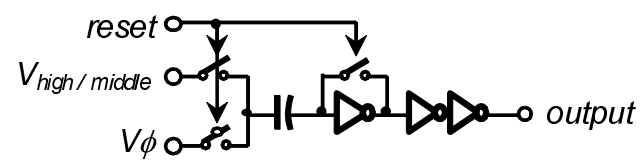

(b)

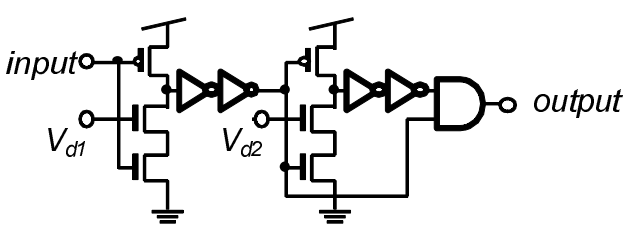

(c)

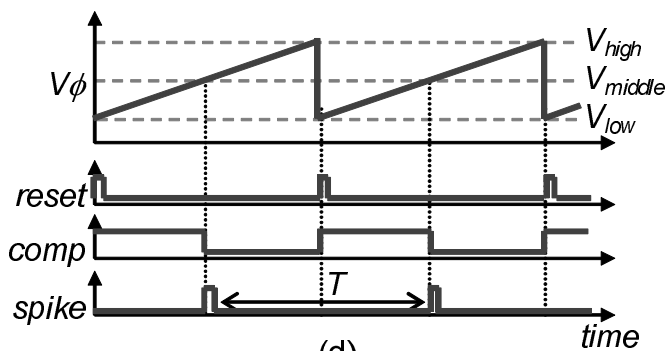

(d)

Fig. 4. Oscillator circuit $O S C$ : (a) oscillator $(O S C)$, (b) comparator (comp),

$(c)$ spike pulse generator $(P G)$, and (d) timing diagram for circuit operation.

threshold voltage $V_{\text {middle }}$, an output spike pulse is generated and transmitted to other oscillators. Then, when $V_{\phi}$ reaches another constant threshold voltage $V_{h i g h}$, signal reset is generated, and $V_{\phi}$ is reset at the lowest voltage $V_{\text {low }}$.

\subsection{Phase sensitivity function generator}

We use binary functions shown in Fig. 5(a) and (b) as function $Z(\phi)$, which approximates $\pm \sin (\phi)$. The phase sensitivity function generator $Z G E N$ is shown in Fig. 5(c), and the timing diagram is shown in Fig. 5(d). The circuit generates signals $Z_{p}$ and $Z_{n}$, which represents function $Z(\phi)$ in the time domain, using received spike pulses, a comparator output, and an external signal sign that determines the function type of $Z$. Signals $Z_{p}$ and $Z_{n}$ represent positive and negative parts of function $Z$, respectively. By combining these two signals, we can generate "- sin"-type function $Z^{-}$and "+ $\operatorname{sin"-type~function~} Z^{+}$. It is noted that a zero-value time span (gap), $\alpha$, exists between non-zero time spans.

\subsection{Phase state updater}

Phase state updater $U P D$ is shown in Fig. 6. The circuit updates $V_{\phi}$ with currents from the switched current sources. The current flows only during the time span corresponding to the pulse width of the spike pulse, $\Delta t$, received from the other oscillators. When a spike pulse is fed into $U P D$, only if $Z_{p}$ is "High", the charging side of the current source $I_{c+}$ is active, and $V_{\phi}$ increases; on the other hand, only if $Z_{n}$ is "High", the discharging side of the current source $I_{c-}$ is active, and $V_{\phi}$ decreases.

It is noted that because $\Delta t$ is finite, the slope of a change in $V_{\phi}$ is also finite. This means that $Z(\phi)$ is not binary but actually piecewise-linear, as shown in Fig. 6(b). In this circuit configuration, we can increase the connections by the number of UPD connected to $O S C$. 


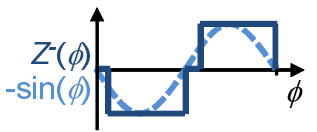

(a)

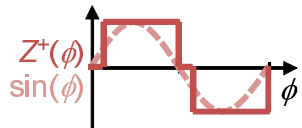

(b)

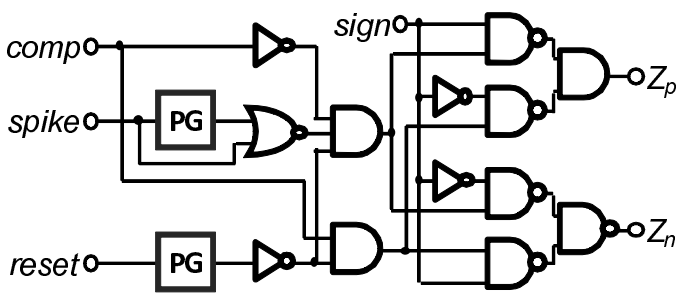

(c)

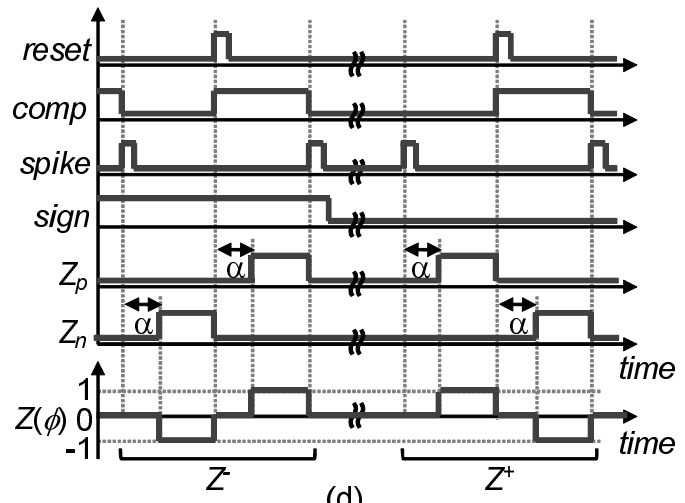

(d)

Fig. 5. Phase sensitivity function generator $Z G E N$ : (a) "- sin"-type function $Z^{-}$, (b) "+ sin"-type function $Z^{+}$, (c) circuit configuration, and (d) timing diagram for generating functions $Z^{ \pm}$.

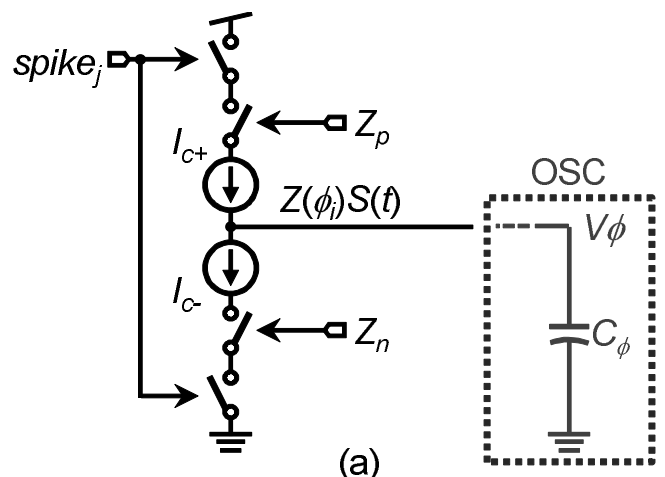

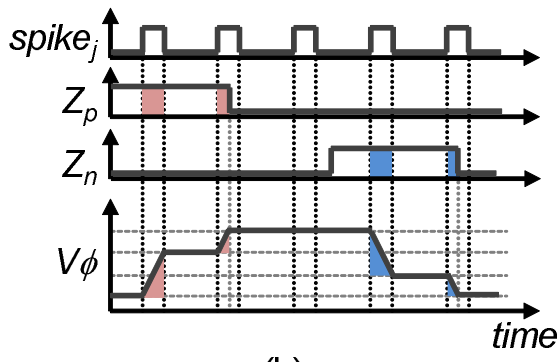

(b)

Fig. 6. Phase state updater $U P D$ : (a) circuit configuration and (b) timing diagram for update operation.

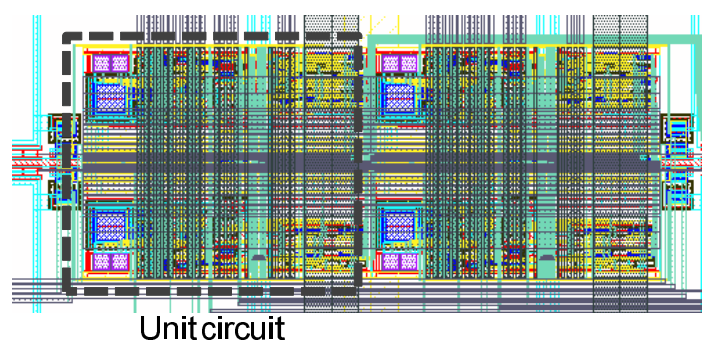

(a)

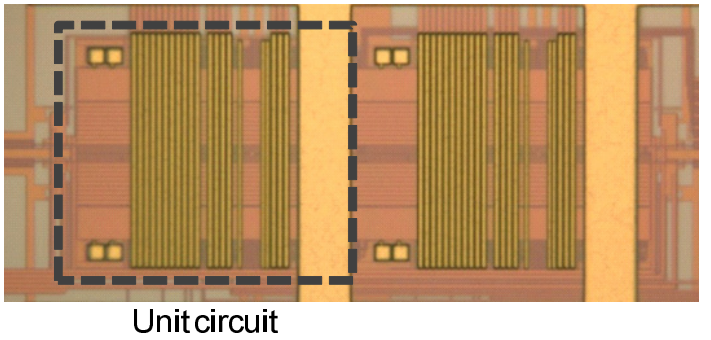

(b)

Fig. 7. Circuit design result: (a) layout result and (b) microphotograph of two pulse-coupled oscillator unit circuits.

\subsection{LSI circuit design}

We designed and fabricated a CMOS circuit in which two unit circuits are connected each other using TSMC $0.25 \mu \mathrm{m}$ (1-Poly, 5-Metal) CMOS technology, in order to construct a system of two pulsecoupled oscillators. The layout result and the microphotograph of the core part of the fabricated circuit are shown in Fig. 7, and the circuit specification is shown in Table I.

\section{Measurement results and discussion}

\subsection{Observation of synchronization states}

Measurement results for this pulse-coupled phase oscillator system are shown in Fig. 8. We set mean firing period $T=1 \mu \mathrm{s}, \Delta t=20 \mathrm{~ns}, \alpha=50 \mathrm{~ns}, V_{\text {high }}=2 \mathrm{~V}, V_{\text {middle }}=1.5 \mathrm{~V}, V_{\text {low }}=1 \mathrm{~V}$, and updating current $I_{c}$ is set about $1 \mu \mathrm{A}$. As shown in Fig. 8(A), if we used function $Z^{-}$and set $\Delta t \leq \alpha<\pi / 2$, two oscillators synchronized with in-phase, where the initial phase difference was $0.95 \pi$. In contrast, if we used function $Z^{+}$and set $\alpha$ as the same condition, they synchronized with anti-phase, where the initial 
Table I. Circuit specification.

\begin{tabular}{ll}
\hline Technology & TSMC 0.25 $\mu \mathrm{m}$ 1-Poly 5-Metal CMOS \\
Layout area & $400 \times 150 \mu \mathrm{m}^{2}$ \\
Supply voltage & $3.3 \mathrm{~V}$ \\
Update period & $1 \mu \mathrm{s}$ \\
Power consumption & $840 \mu \mathrm{W}$ (estimated by SPICE) \\
\hline
\end{tabular}

phase difference was $0.05 \pi$, as shown in Fig. 8(B). In addition to those, if we used functions $Z^{-}$and $Z^{+}$with $\alpha>\pi / 2$, we found other synchronization states, as shown in Fig. 8(C) and (D), respectively. This different-phase (out-of-order) synchronization was indicated in another literature [16], where the synchronization occurred due to time delay of spike pulses. On the other hand, in our circuit, the synchronization is induced by a certain value of parameter $\alpha$, as described below.

Synchronization mechanisms for the four cases shown in Fig. 8 are explained using Fig. 9 as follows.

Regarding in-phase synchronization case (A), if the initial state is in about an anti-phase condition, as shown in Fig. 9(A)(a), $\phi_{j}$ leads because $Z_{j}$ is positive when a spike pulse from oscillator $i$ reaches oscillator $j$, and $\phi_{i}$ lags because $Z_{i}$ is negative when a spike pulse from oscillator $j$ reaches oscillator $i$. Then, two oscillators reach the state shown in Fig. 9(A)(b), where one oscillator (oscillator $i$ in this case) is not updated because of gap $\alpha$, but the other is still updated. Finally, the system reaches the steady state shown in Fig. 9(A)(c). If some fluctuation occurs and synchronization is broken, the system goes back to the state Fig. 9(A)(b), and then reaches the steady state again. Thus, this system has stability to stay at the in-phase synchronization state.

Regarding anti-phase synchronization case (B), if the initial state is in about an in-phase condition, as shown in Fig. 9(B)(a), one oscillator ( $j$ in this case) is updated, and $\phi_{j}$ lags because $Z_{j}$ is negative when a spike pulse from oscillator $i$ reaches oscillator $j$. On the other hand, oscillator $i$ is not updated because of gap $\alpha$, Then, the phase difference $\Delta \phi$ becomes large, and the system reaches the state shown in Fig. 9(B)(b), where both oscillators are updated and $\Delta \phi$ becomes larger. Finally, the system reaches the steady state shown in Fig. 9(B)(c). In the similar way to case (A), this system also has stability to stay at the anti-phase synchronization state.

Figure $9(\mathrm{C})$ shows a different-phase synchronization state when using function $Z^{-}$. As in case (A), the initial state is in about an anti-phase condition, as shown in Fig. 9(C)(a). One oscillator $(i$ in this case) is updated, and $\phi_{i}$ lags because $Z_{i}$ is negative when a spike pulse from oscillator $j$ reaches oscillator $i$. Then, the system reaches the state shown in Fig. 9(C)(b), where both oscillators are not updated because $\alpha>\pi / 2$ and both spike pulses are in gap $\alpha$. If some fluctuation occurs and $\Delta \phi$ becomes larger, the system still stay at the same state, but if $\Delta \phi$ becomes smaller, and state shown in Fig. 9(C)(c) is realized, the system reaches the in-phase synchronization state.

Figure $9(\mathrm{D})$ shows another different-phase synchronization state when using function $Z^{+}$. As in case (B), the initial state is in about an in-phase condition, as shown in Fig. 9(D)(a). One oscillator $(j$ in this case) is updated, and $\phi_{j}$ lags because $Z_{j}$ is negative when a spike pulse from oscillator $i$ reaches oscillator $j$. Then, the system reaches the state shown in Fig. 9(D)(b), where both oscillators are not updated as in the state shown in Fig. 9(C)(b). If some fluctuation occurs and $\Delta \phi$ becomes smaller, the system still stay at the same state, but if $\Delta \phi$ becomes larger, and state shown in Fig. 9(D)(c) is realized, the system reaches the anti-phase synchronization state.

\subsection{Measurement results about settling time}

We investigated the relationship between updating current $I_{c \pm}$ and the settling time until synchronization. Circuit simulation (HSPICE) results about the relationship between the number of spikes and phase-difference $\Delta \phi$ in transition states toward the in-phase synchronization state are shown in Fig. 10(a) as a parameter of $I_{c}\left(=I_{c \pm}\right)$. The simulation condition is the same as that described in 4.1. Measurement results using the fabricated circuit about spike trains in the same transition states are shown in Fig. 10(b). The convergence timing in each condition is marked by a red circle in Fig. 10(b), and they are nearly identical with the simulation results shown in Fig. 10(a). Each update amount is proportional to charges updated in capacitor $C_{\phi}$, as shown in Fig. 6(a), and it is given by $I_{c} \cdot \Delta t$. 


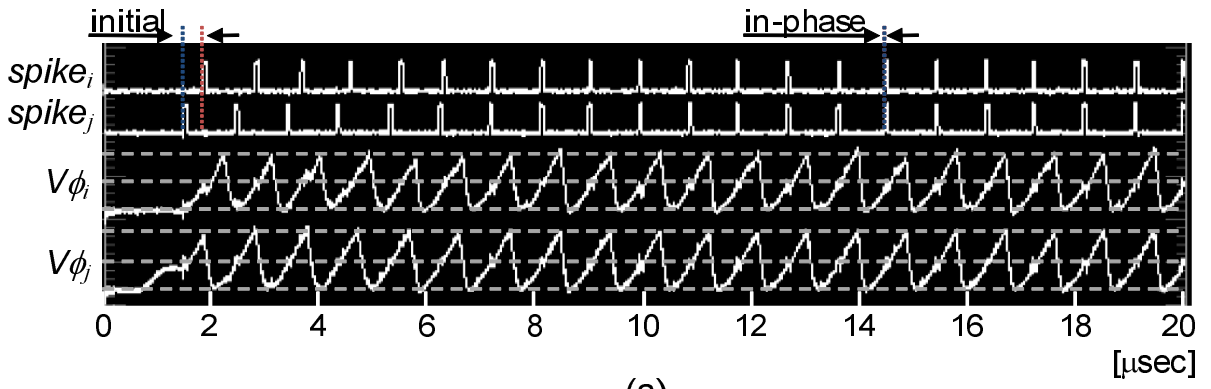

(a)

(A)

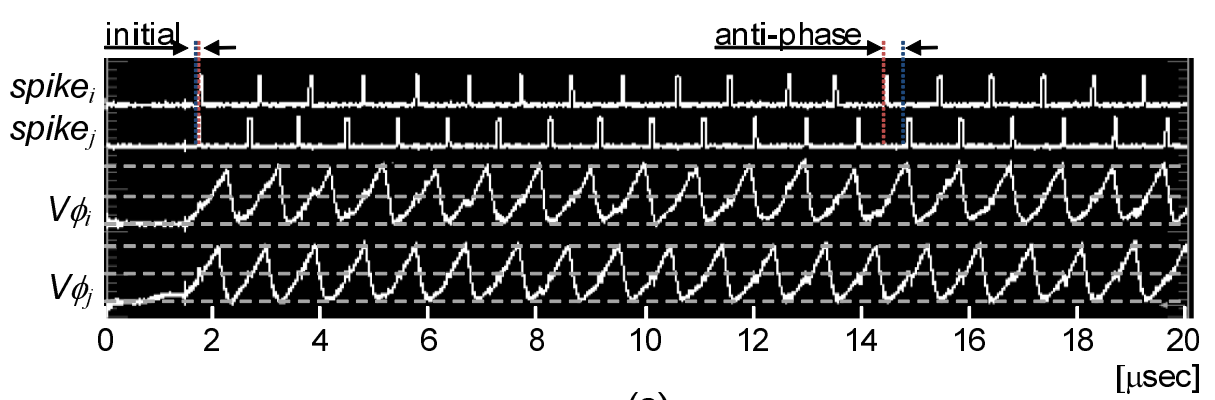

(a)

(B)

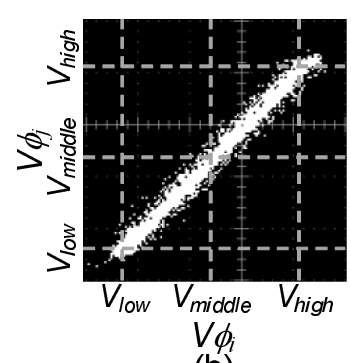

(b)

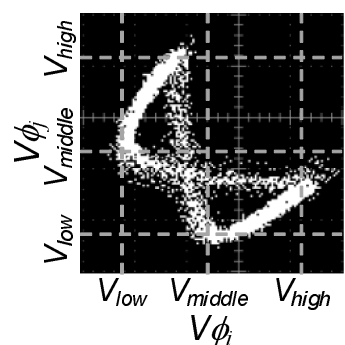

(b) (a)

(C)

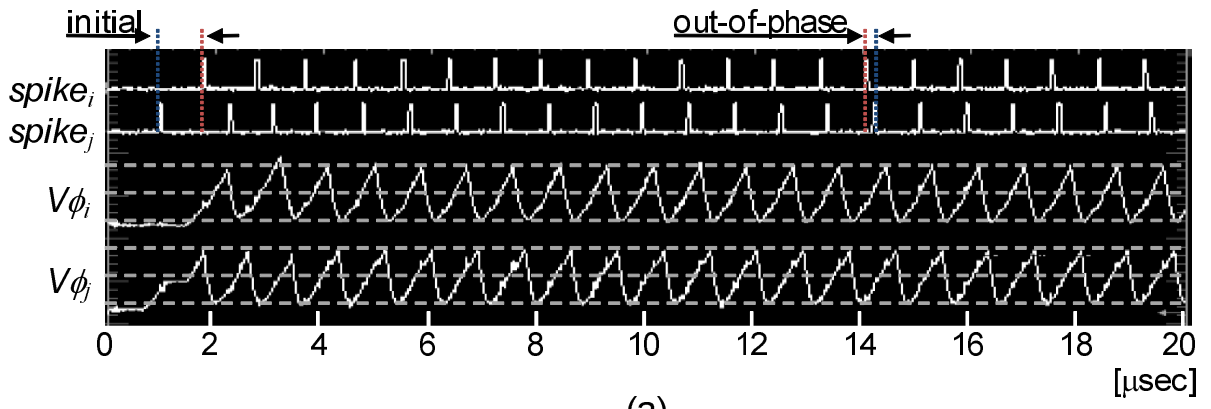

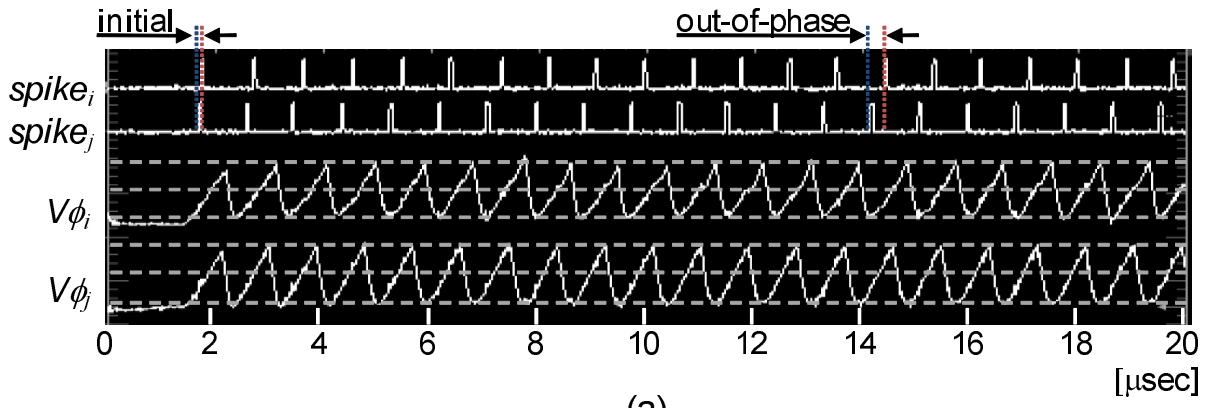

(a)

(D)

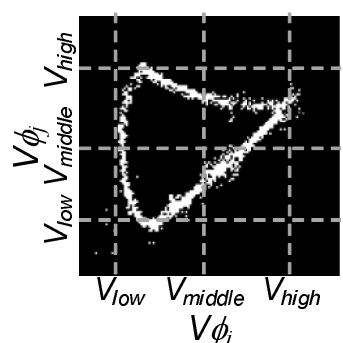

(b)

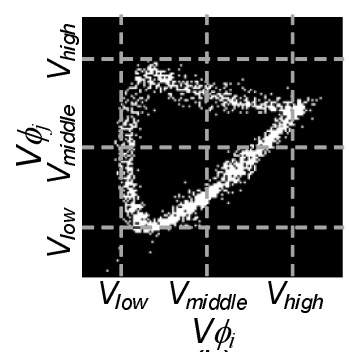

(b)

Fig. 8. Measurement results of synchronization states in the CMOS pulsecoupled phase oscillator system: (A) in-phase state (phase-difference $\Delta \phi=0$, function $\left.Z^{-}\right)$, (B) anti-phase state $\left(\Delta \phi=\pi\right.$, function $\left.Z^{+}\right),(\mathrm{C})$ different-phase state $\left(\Delta \phi \neq 0, \pi\right.$, function $\left.Z^{-}\right)$, and (D) different-phase state $(\Delta \phi \neq 0, \pi$, function $Z^{+}$): (a) observed waveforms and (b) $V_{\phi_{i}}-V_{\phi_{j}}$ plot.

Therefore, $\Delta \phi$ is expected to change in proportion with $I_{c}$, and equivalently the number of spikes required to reach a steady state, $N_{c o n v}$, is inversely proportional to $I_{c}$, as shown in Fig. 6(c).

It can also be found from these measurement results that the convergence time to reach synchronization states in our pulse-coupled oscillator system is much shorter than that in the phase-coupled phase oscillator system that we previously proposed [6], in which the convergence time was more than $200 \mu \mathrm{s}$ (100 time step). 


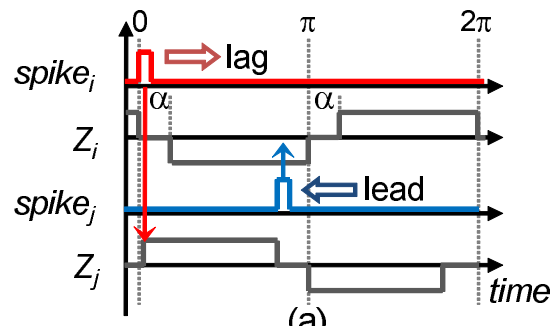

(a)

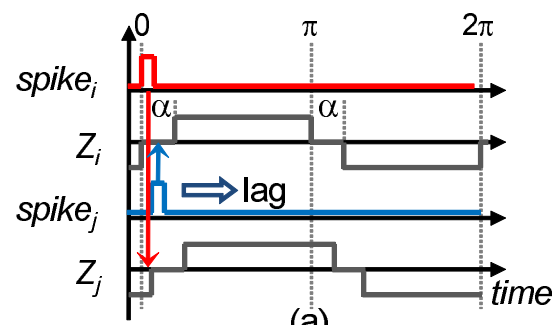

(a)

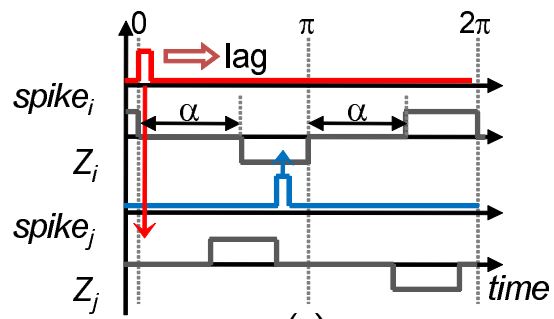

(a)

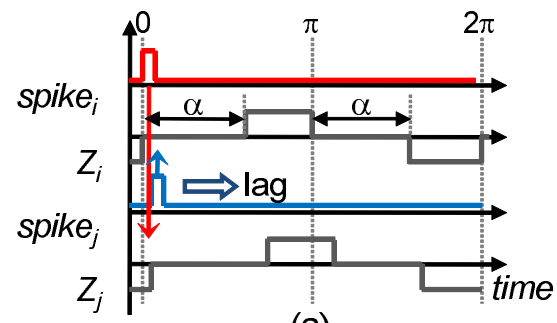

(a)

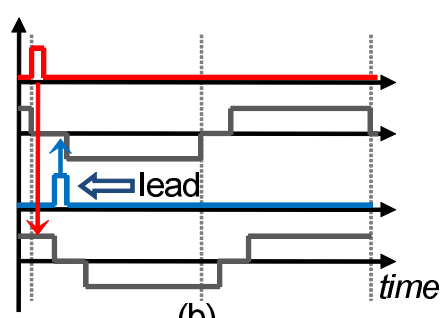

(A)

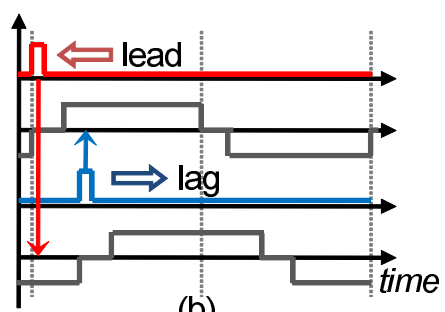

(B)

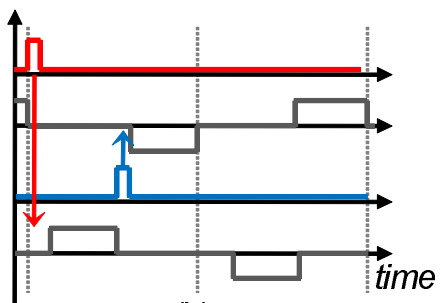

(b)

(C)

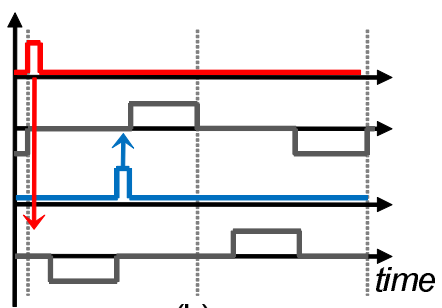

(b)

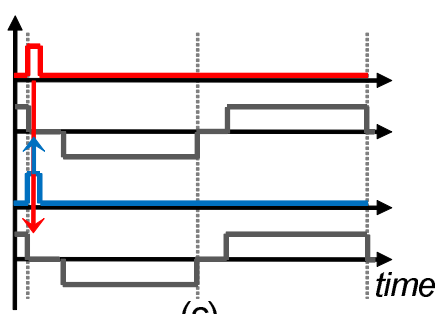

(c)

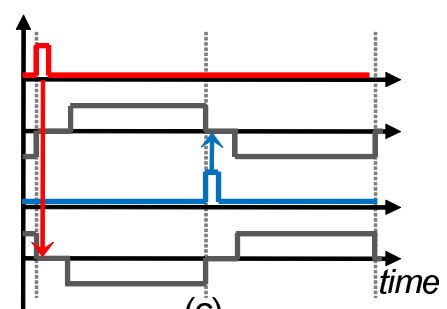

(c)

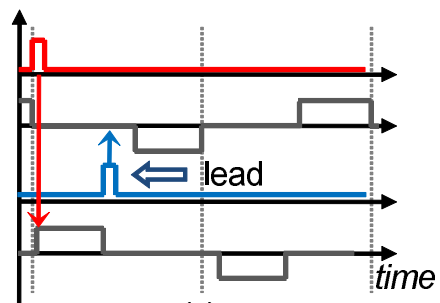

(c)

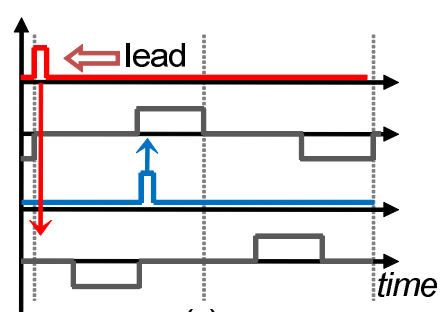

(c)

(D)

Fig. 9. Synchronization mechanisms: (A) to (D) correspond to those in

Fig. 8, and (a) initial state, (b),(c) transient state or steady state.

Other circuit simulation results are shown in Fig. 11, where $\alpha=\Delta t=20$ ns and other conditions are same as in Fig. 10(a). Period-2 oscillations are observed in synchronization states at large update amounts. This is because update operation is performed in the time span caused by delays in spike pulse transmission between oscillators and by operation delays in circuits $O S C$ and $Z G E N$. On the other hand, such period-2 oscillations are not observed in Fig. 10(a). From these results, we can conclude that the effect of transmission/operation delays can be eliminated by setting $\alpha>\Delta t$. This feature is also effective to compensation for calculation precision degradation due to CMOS device mismatches and other nonidealities in analog circuits. In our measurement, timing fluctuation of about $1.5 \mathrm{~ns}$ was observed at a mean firing period of $1 \mu \mathrm{s}$. This nonideality was successfully compensated by the setting regarding $\alpha$, and steady synchronization behavior was observed.

\section{Conclusion}

We proposed an analog CMOS circuit for a system of pulse-coupled oscillators. The circuit was 


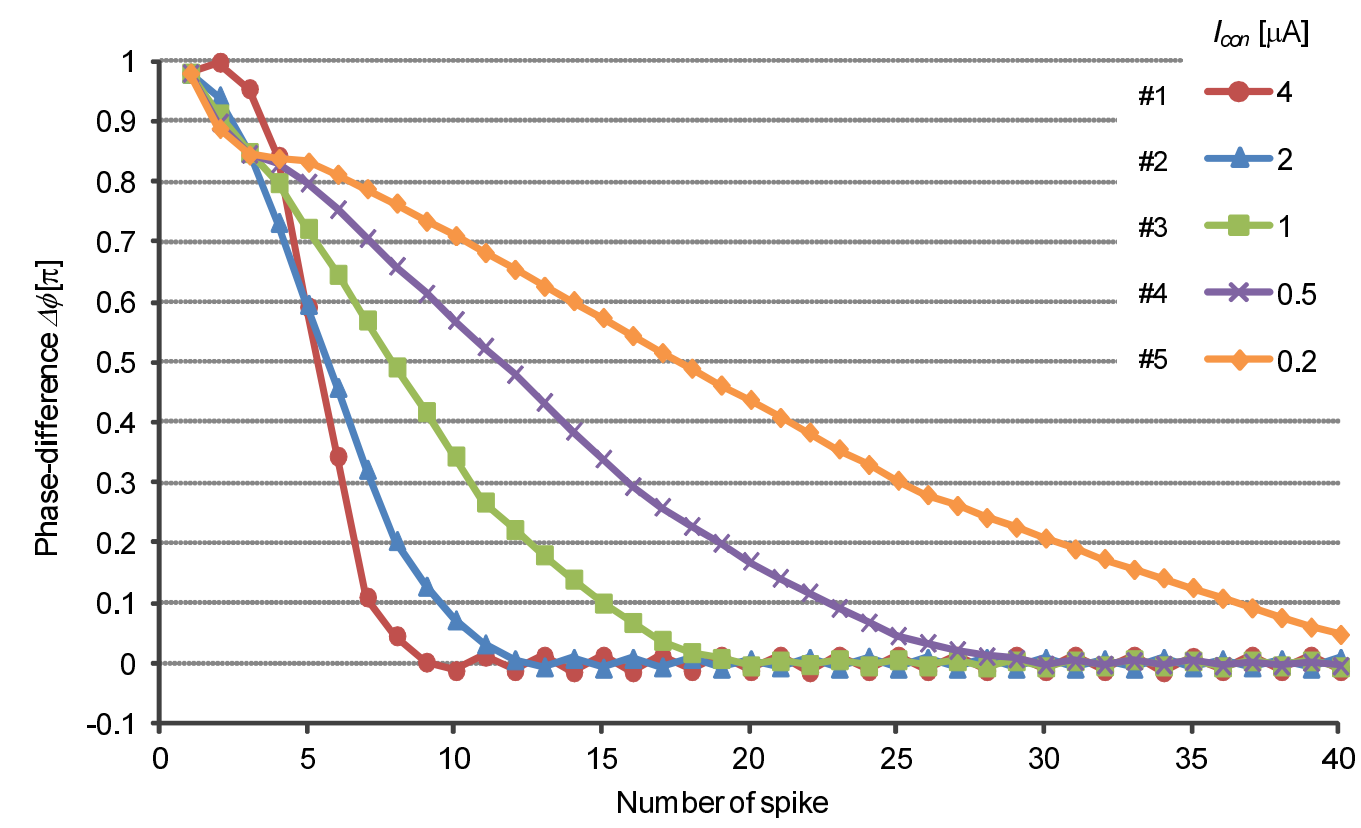

(a)

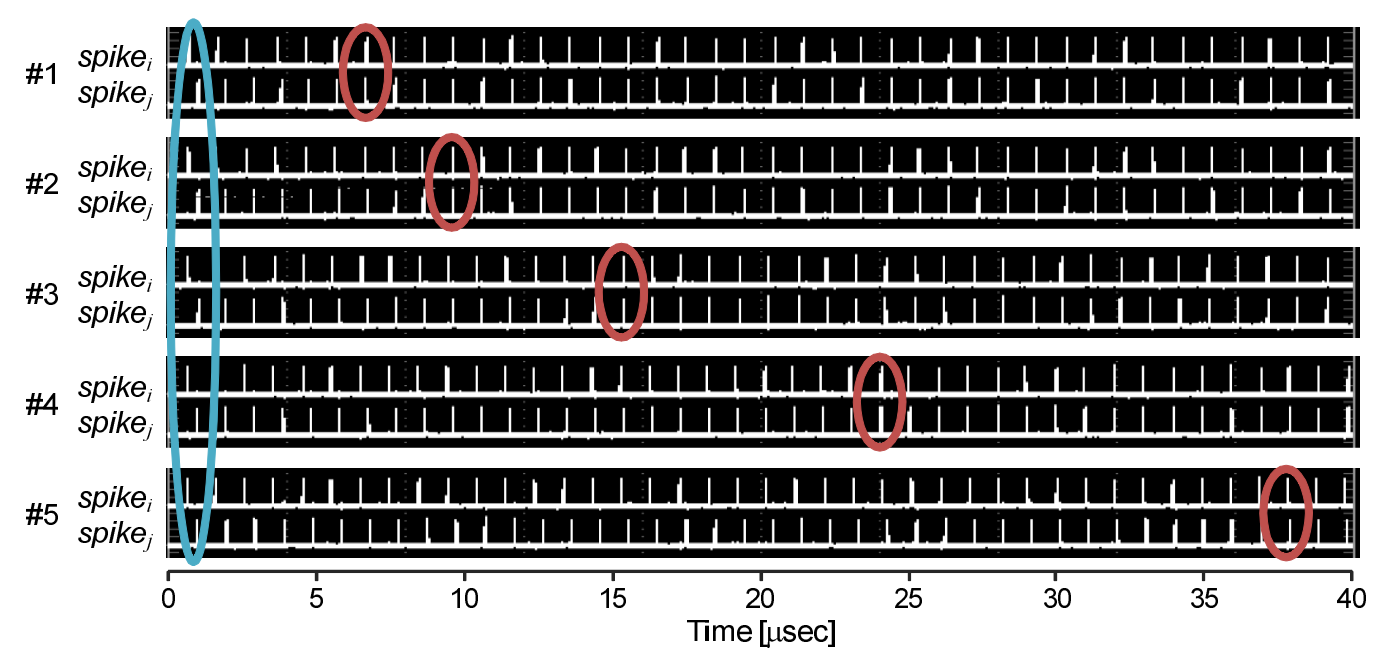

(b)

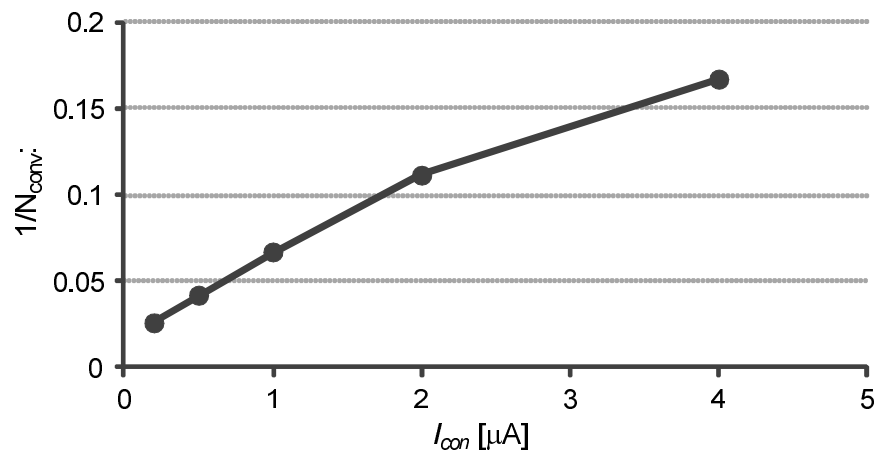

(c)

Fig. 10. Relationship between updating amount and settling time until synchronization where $\Delta t=20 \mathrm{~ns}, \alpha=50$ ns: (a) circuit simulation (HSPICE) results, (b) circuit measurement results, and (c) relationship between $I_{c}$ and $1 / N_{\text {conv }}$.

designed and fabricated using a $0.25 \mu \mathrm{m}$ CMOS technology. We observed in/anti-phase and other types of synchronization states in the measurement using the fabricated chip. We also found that such synchronizations occur very quickly because of instantaneous updating with spike timing, which will 


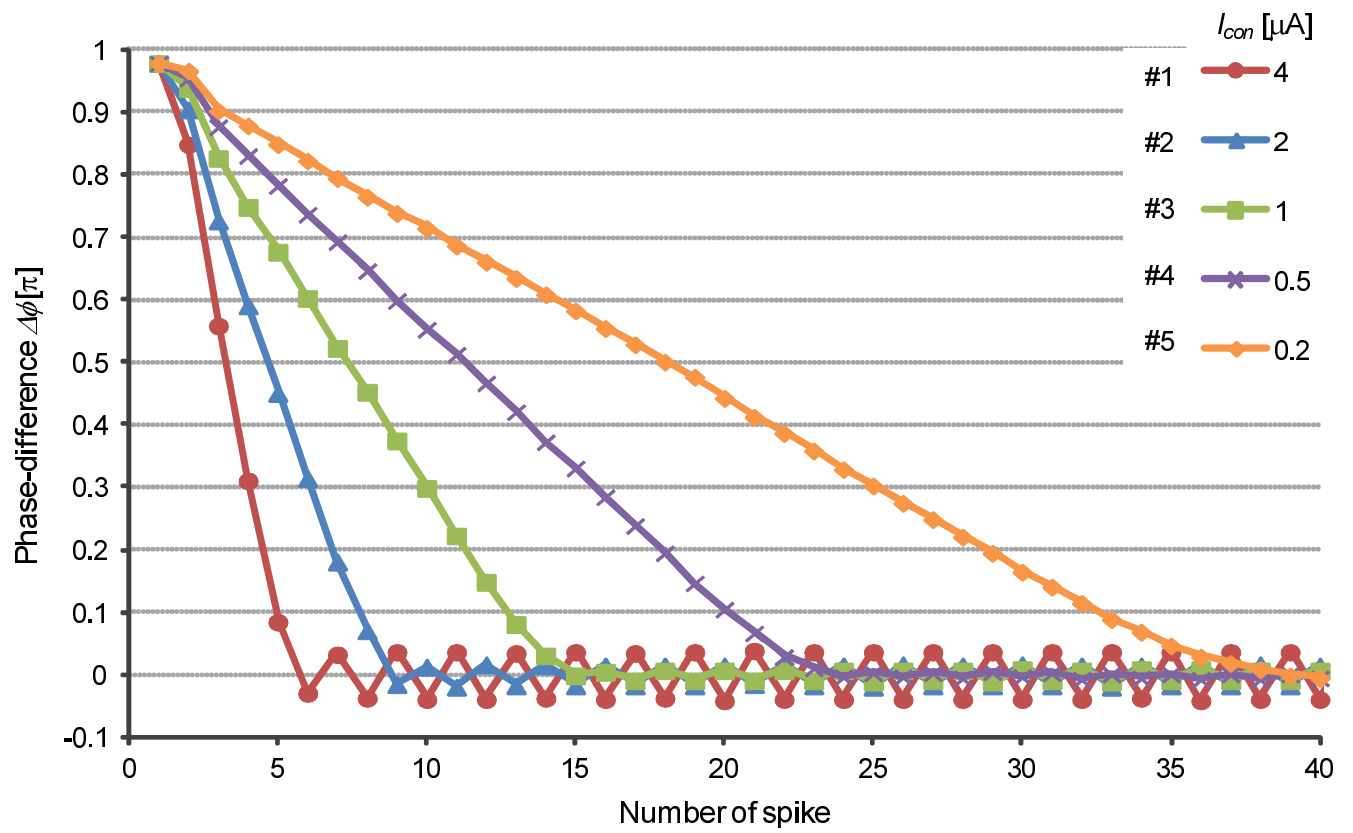

Fig. 11. Circuit simulation (HSPICE) results of relationship between updating amount and settling time until synchronization, where $\alpha=\Delta t=20 \mathrm{~ns}$ ).

lead to high-speed, highly-effective spike-based computing systems. A promising application of such systems is image processing, and integrated circuit design for large-scale networks of pulse-coupled oscillators will be expected as future work.

\section{Acknowledgments}

This work was partly supported by Grant-in-Aid for challenging Exploratory Research \#23650118, and the Aihara Project, the FIRST program from JSPS, initiated by CSTP. The LSI chip design was supported by VLSI Design and Education Center (VDEC), the University of Tokyo in collaboration with Cadence Design Systems, Inc.

\section{References}

[1] Y. Kuramoto, "Collective synchronization of pulse-coupled oscillators and excitable units," Physica D, vol. 50, no. 1, pp. 15-30, 1991.

[2] Y. Kuramoto, Chemical Oscillation, Waves, and Turbulence, Springer, Berlin, 1984.

[3] A.T. Winfree, The Geometry of Biological Time, Springer, New York, 1980.

[4] F.C. Hoppensteadt and E.M. Izhikevich, Weakly Connected Neural Networks, Springer, New York, 1997.

[5] E.M. Izhikevich, Dynamical Systems in Neuroscience: The Geometry of Excitability and Bursting, MIT Press, Cambridge, MA, 2007.

[6] D. Atuti, N. Kato, K. Nakada, and T. Morie, "CMOS circuit implementation of a coupled phase oscillator system using pulse modulation approach," European Conf. on Circuit Theory and Design (ECCTD), pp. 827-830, August 2007.

[7] T. Morie, K. Murakoshi, M. Nagata, and A. Iwata, "Pulse modulation techniques for nonlinear dynamical systems and a CMOS chaos circuit with arbitrary 1-D maps," IEICE Trans. Electron., vol. E87-C, no. 11, pp. 1856-1862, 2004.

[8] D. Atuti, K. Nakada, and T. Morie, "CMOS pulse-modulation circuit implementation of phaselocked loop neural networks," IEEE Int. Symp. on Circuits and Systems (ISCAS), pp. 21742177, May 2008.

[9] Y. Kawashima, D. Atuti, K. Nakada, M. Okada, and T. Morie, "Coarse image region segmentation using region- and boundary-based coupled MRF models and their PWM VLSI implementation," Proc. Int. Joint Conf. on Neural Networks (IJCNN), pp. 1559-1565, June 2009. 
[10] K. Matsuzaka, K. Nakada, and T. Morie, "Analog CMOS circuit implementation of a system of pulse-coupled oscillators for spike-based computation," IEEE Proc. of Int. Symp. Circuits and Systems (ISCAS), pp. 2849-2852, May 2011.

[11] X.Y. Wang and A. Apsel, "Pulse coupled oscillator synchronization for low power UWB wireless transceivers," Proc. 50th Midwest Symposium on Circuits and Systems, pp. 1524-1527, August 2007.

[12] X.Y. Wang, R. Dokania, and A. Apsel, "PCO based synchronization for ad-hoc duty-cycled impulse radio sensor networks," Special issue on cognitive sensor networks of IEEE Sensors Journal, vol. 11, no. 3, pp. 555-564, 2011.

[13] X.Y. Wang, R.K. Dokania, and Y. Zhuang, C.I. Dorta-Quinones, W. Godycki, M. Lyons, and A.B. Apsel, "A self-synchronized, crystal-less, $86 \mu \mathrm{W}$, dual-band impulse radio for ad-hoc wireless networks," IEEE RFIC Symposium, pp. 5-10, June 2011.

[14] K. Matsuzaka and T. Morie, "A simplified region-based coupled MRF model for coarse image region segmentation toward its VLSI implementation," Proc. of Int. Symp. on Nonlinear Theory and Its Applications (NOLTA), pp. 202-205, October 2009.

[15] R.E. Mirollo and S.H. Strogaz, "Synchronization of pulse coupled oscillators," SIAM J. Appl. Math., vol. 50, pp. 1645-1662, 1990.

[16] V.V. Klinshov and V.I. Nekorkin, "Synchronization of time-delay coupled pulse oscillators," Chaos, Solitons \& Fractals, vol. 44, no. 1-3, pp. 98-107, 2011. 